\title{
A Novel Fabrication Method for All-Fiber, Weakly Fused, Polarization Beamsplitters
}

\author{
Cheng Wen Wu, Tzong-Lin Wu and Hung-Chun Chang, Member, IEEE
}

\begin{abstract}
A simple method for fabricating single-mode fused couplers for polarization beamsplitting is presented. The coupler is extremely weakly fused such that the first polarization modulation waist in its power transfer characteristic is clearly extended. Due to such a feature, the coupler drawing process may accurately be stopped at the waist point by simply monitoring the power variations of the output ports. We have achieved an average extinction ratio of $-23.7 \mathrm{~dB}$ at the throughput port and $-23.8 \mathrm{~dB}$ at the coupled port at the designated wavelength. The polarization bandwidth obtained is $38 \mathrm{~nm}$.
\end{abstract}

\section{INTRODUCTION}

$\mathbf{F}$ IBER-OPTIC polarization beamsplitters (PBS) made with standard communication fibers can be used to separate and combine two orthogonal polarized lights, and have applications in sensor and coherent communication systems. These devices were first fabricated by drawing very long couplers [1], [2]. The couplers underwent many power transfer cycles during such a very long drawing process. The difficulty and the more complicated fabrication procedure required have been described by Eisenmann and Weidel [3]. They measured the degree of polarization (DOP) of the light from output fibers during fabrication and finished the coupler drawing process at the maximum of the DOP. More recently, Morishita and Takashina [4] reported detailed experimental studies of the polarization properties of fused couplers and characteristics of the PBS's under different fusing conditions. Since the PBS's in the above reports were all achieved after drawing through many power transfer cycles, the power transfer variation versus the drawn length became fast and the stop point in the drawing process was critical or difficult to control. Fabrication of a high-quality PBS was therefore difficult. Moreover, the PBS thus made had a narrow bandwidth (17 $\mathrm{nm}$ in [3]). In this letter, we present a simple method to overcome the above-mentioned difficulty. The coupler is extremely weakly fused such that the tapered fibers are nearly touching in the coupling region. Consequently, the number of power transfer cycles is greatly reduced, and the first PBS point can easily be determined.

Manuscript received January 20, 1995; revised March 10, 1995. This work was supported in part by the National Science Council of the Republic of China under Grants NSC81-0417-E002-01 and NSC82-0417-E002-222 and in part by Telecommunications Laboratories, Ministry of Communications, Republic of China, under Grants TL-NSC-81-5103 and TL-NSC-82-5106.

C. W. Wu and T,-L. Wu are with Department of Electrical Engineering, National Taiwan University. Taipei, Taiwan 106-17, Republic of China.

H.-C. Chang is with the Department of Electrical Engineering and the Graduate Institute of Electro-optical Engineering, National Taiwan University,

Taipei, Taiwan 106-17, Republic of China.

IEEE Log Number 9411733.

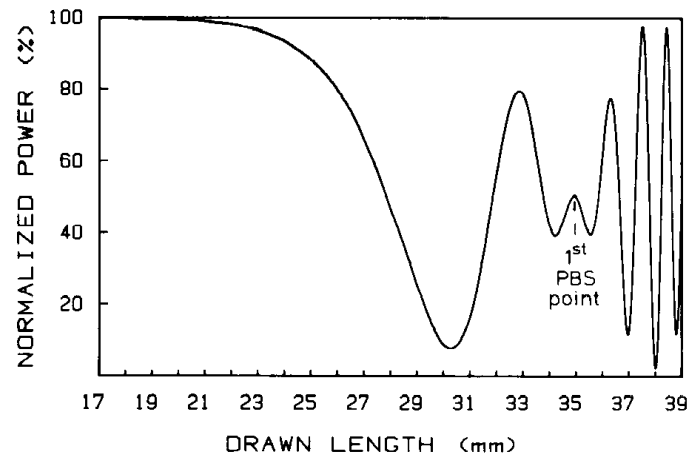

Fig. 1. Power transfer characteristic versus the drawn length at the throughput port of the extremely weakly fused coupler measured with an unpolarized 1523-nm He-Ne laser.

\section{BASIC PRINCIPLE}

Due to the form birefringence of the fused coupler, $x$ and $y$ polarized lights have different power transfer phases after an interaction length $S$. The variation of the coupler's output power as a function of the drawn length would reveal a polarization modulation characteristic [1], [2]. When the difference between the power transfer phases equals $\pm \pi / 2$ at a particular interaction length $S_{\pi / 2}$, the first polarization modulation waist point (i.e., the PBS point as shown in Fig. 1) occurs with the $x$ polarized light appearing at one output port and the $y$ polarized light at the other. The interaction length $S_{\pi / 2}$ is influenced by the magnitude of the form birefringence. Larger form birefringence results in smaller $S_{\pi / 2}$. In addition, lesser coupling strength between the fibers would correspond to a larger power oscillation period in the drawing process. If both $S_{\pi / 2}$ and the coupling strength between the fibers are small enough, there would exist only a few power transfer cycles before the coupler's first PBS point occurs. Due to the large oscillation period in the first few power transfer cycles, the coupler's first polarization waist would become so extended that the PBS point is clear and thus easily determined. According to our previous simulation studies, the extremely weakly fused couplers (with touching fibers) would have large form birefringence and small coupling strength such that the PBS operation could occur within a few power transfer cycles [5]. Therefore, a suitable method for fabricating PBS's seems to be the one based on extremely weakly fused couplers.

\section{DEVICE FABRICATION}

For the purpose of making extremely weakly fused couplers, an electric heater made of electric resistance wires was 


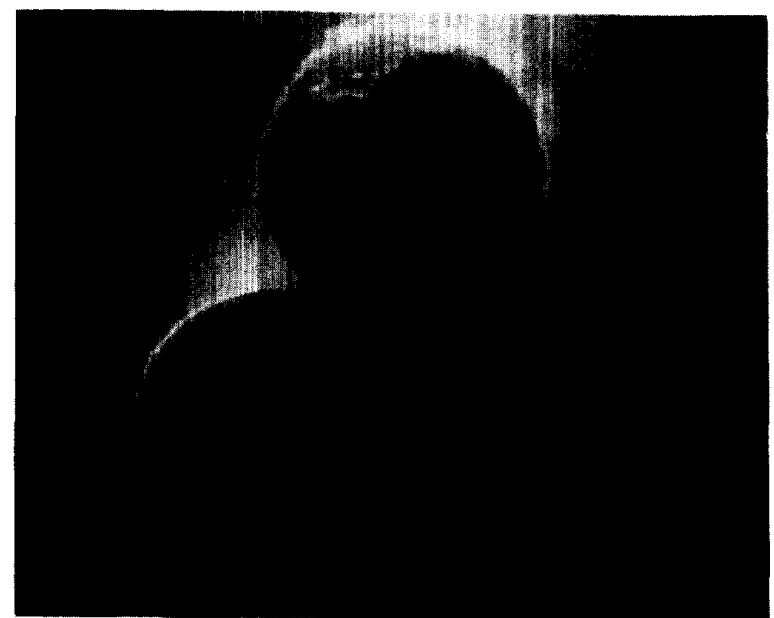

Fig. 2. SEM photograph of the cross-sectional shape of the extremely weakly fused PBS. The measured aspect ratio is 1.98 .

designed. Its measured peak temperature was about $1200{ }^{\circ} \mathrm{C}$. The standard communication fibers (Newport F-SS) prepared to manufacture the coupler were woven together before being moved into the heater for fusing so that they would not be separated from each other under weak fusing conditions. The heater made longitudinal oscillations in the drawing process in order to increase the heated length of fibers. The fiber drawing speed was $49 \mu \mathrm{m} / \mathrm{s}$. The oscillation period of the heater was $4 \mathrm{~mm}$ with a moving speed of $80 \mu \mathrm{m} / \mathrm{s}$. An unpolarized $1523-\mathrm{nm} \mathrm{He}-\mathrm{Ne}$ laser was used to monitor the power transfer behavior of the coupler.

The typical power transfer characteristic of the extremely weakly fused coupler measured at the throughput port is shown in Fig. 1. The extended first polarization modulation waist is observed. The clear first PBS point just occurs at the fourth extremum of the curve. Based on this characteristic, a procedure for manufacturing PBS's has been developed. The two output powers of the coupler were simultaneously measured and their ratios were calculated. When the ratio was equal to 0.5 , a defined parameter $N$ was increased by one. After $N=3$ and an additional time delay, the stop action was initiated. Four PBS's have been successively fabricated by following this procedure. After being packaged and measured with a rotatory polarizer at the output port, the average polarization extinction ratios of these PBS's were $-23.7 \mathrm{~dB}$ and $-23.8 \mathrm{~dB}$ at the throughput port and the coupled port, respectively, for the unpolarized $1523-\mathrm{nm}$ light. The average excess loss measured with the cut-back method was $0.2 \mathrm{~dB}$. Note that only output powers are monitored in our method. The measurement of DOP using a more complex polarization modulator as in [3] is not required here. The fabrication of the PBS becomes easy as in the fabrication of usual power dividers.

\section{DISCUSSION}

The large average polarization extinction ratio obtained in our method is attributed to the noncritical behavior of the

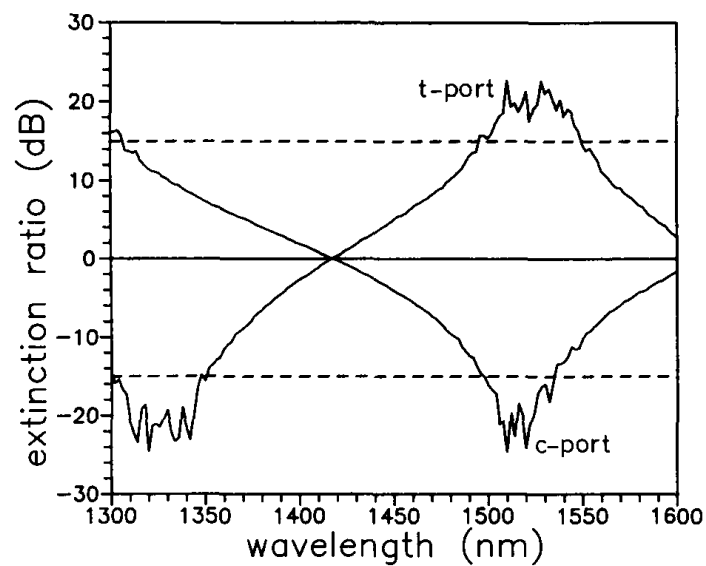

Fig. 3. Polarization spectral response characteristics of the extremely weakly fused PBS at the throughput port and the coupled port.

first PBS point. As Fig. 1 shows, the power transfer period including the PBS point is $1.3 \mathrm{~mm}$, being larger than that in the previous method [3]. For a more strongly fused coupler, the period was only about $0.1 \mathrm{~mm}$ [3]. A large period relaxes the severe stopping condition of the PBS. High-quality PBS's can easily be fabricated by simply monitoring the powers from the output fibers as in fabricating usual power-dividing couplers. The power transfer period may further be made larger by reducing the peak temperature of the electric heater. The coupler would have smaller coupling strength if it were fused more weakly at lower temperatures. But, owing to the high viscosity of the fiber at low temperatures, the coupler is easily broken in the drawing process. Fig. 2 shows the SEM (scanning electron microscope) photograph of the crosssectional shape of one of our PBS's cut at the neck region. The measured aspect ratio is 1.98 (note that the aspect ratio is 2.0 for the touching-fiber case), showing that it is indeed an extremely weakly fused coupler.

Fig. 3 shows the measured spectral responses of the polarization extinction ratio for the extremely weakly fused PBS at the two output ports. The polarization measurements were made by first searching the two orthogonal birefringent axes of the device with polarized light from a monochromator. Then calculation of the extinction ratio at each wavelength for each of the two output ports was accomplished by measuring the transmission of the light polarized along each of the two axes. As Fig. 3 shows, the polarization extinction ratios around the 1523-nm wavelength at the two output ports are both larger than $16 \mathrm{~dB}$, showing that the coupler was indeed stopped at the PBS point. The polarization bandwidth defined by the bandwidth of the extinction ratio larger than $15 \mathrm{~dB}$ is $38 \mathrm{~nm}$, which is more than twice that reported in [3]. The wider bandwidth is attributed to the slower variation of the power transfer curve around $S_{\pi / 2}$. For 1320-nm light, the device has a large extinction ratio at the throughput port, but not at the other port. This is due to the fact that the coupling strength is wavelength-dependent such that the transmission of one polarized light is zero at the throughput port but the other polarized light is not zero at the coupled port. 


\section{CONCLUSION}

A novel method for fabricating single-mode fused biconical polarization beamsplitters based on an extremely weakly fused coupler structure has been proposed. The fibers in the fused region are nearly touching. Before reaching the first PBS point during the drawing process, there are only few power transfer cycles, due to a large power transfer period. Such features lead to a noncritical first PBS point, facilitating accurate stopping of the drawing process by simply monitoring the output power variation. The average extinction ratio of the fabricated PBS's measured at each of the two output ports at the designated wavelength is better than $-23 \mathrm{~dB}$, with a $38-\mathrm{nm}$ bandwidth.

\section{REFERENCES}

[1] M. S. Yataki, D. N. Payne, and M. P. Varnham, "All-fiber polarising beamsplitter," Electron. Lett., vol. 21, pp. 249-251, 1985.

[2] T. Bricheno and V. Baker, "All-fiber polarization splitter/combiner," Electron. Lett., vol. 21, pp. 251-252, 1985.

[3] M. Eisenmann and E. Weidel, "Single-mode fused biconical coupler optimized for polarization beamsplitting," J. Lightwave Technol., vol. 9 , pp. $853-858,1991$.

[4] K. Morishita and K. Takashina, "Polarization properties of fused fiber couplers and polarizing beamsplitters," J. Lightwave Technol., vol. 9, pp. 1503-1506, 1991

[5] H. C. Chang and T. H. Lin, "Electromagnetic analysis of fused tapered optical fiber couplers," in Proc. 1992 USRI Int. Symp. Electromagnetic Theory, Sydney, Australia, Aug. 17-20, 1992, pp. 445-447. 